\title{
Structural Health Monitoring for Civil Structures: From the Lab to the Field
}

\author{
Piervincenzo Rizzo, ${ }^{1}$ Yi Qing Ni, ${ }^{2}$ and Jinying $\mathrm{Zhu}^{3}$ \\ ${ }^{1}$ Department of Civil \& Environmental Engineering, Swanson School of Engineering, University of Pittsburgh, \\ Pittsburgh, PA 15260, USA \\ ${ }^{2}$ The Hong Kong Polytechnic University, Kowloon, Hong Kong \\ ${ }^{3}$ Civil, Architectural and Environmental Egnineering Department, University of Texas at Austin, Austin, \\ TX 78712-0273, USA
}

Correspondence should be addressed to Piervincenzo Rizzo, pir3@pitt.edu

Received 11 August 2010; Accepted 11 August 2010

Copyright (c) 2010 Piervincenzo Rizzo et al. This is an open access article distributed under the Creative Commons Attribution License, which permits unrestricted use, distribution, and reproduction in any medium, provided the original work is properly cited.

The field of structural health monitoring (SHM) is rapidly growing to provide robust diagnosis and prognosis of many engineering systems spanning from civil and transportation infrastructures to aerospace and military applications. The development and deployment of a robust SHM scheme is pivotal to ensure the safe and cost-effective operation of any engineering system. SHM is mainly a process of (i) observing or tracing the performance of an engineering structure/system under environmental and operational loads by sensors and instrumentation devices, (ii) evaluating the performance of the structure/system for any development of defect or damage by use of the measured data and analytical tools, and (iii) issuing an alarm when the designated performance criteria are exceeded.

Compared to other engineering fields, the research on SHM is relatively young and therefore most of the work has been conducted at universities or companies that have an interest in research and development.

This special issue of the journal is mainly dedicated to those SHM strategies that after being successfully proven in the laboratory were or are currently deployed in the field for field testing and validation.

This special issue contains twelve research papers and three review articles. The topics dealt with in these papers cover a wide variety of SHM methodologies (in alphabetical order): acoustic emission, electromechanical impedance, fiber optics, guided ultrasonic waves, imaging, time-domain reflectometry, and vibration measurements. These methods are applied to one of the following: (1) detection of damage in an ASCE benchmark structure, composite materials, concrete structures, dikes, precast concrete box girders railroads, and water and wastewater pipelines; (2) evaluation bridge scour; (3) measurement of stress or strain in oil and gas pipes, concrete structures, or cables; (4) detection of low to medium energy impact in wind turbine blades; (5) detection of water leaks; (6) identification of modal parameters; (7) measurement of vibration of high-rise reinforced concrete building.

Thanks are given to the issue's guest editors Dr. Yi Qing Ni from The Hong Kong Polytechnic University and Dr. Jinying Zhu from The University of Texas for help in soliciting papers, keeping track of submissions, reviews, rebuttals, and followups. Also, special thanks go to Dina Attia for help in the organization of the accepted papers and the final production of the special issue.

Piervincenzo Rizzo Yi Qing Ni Jinying Zhu 

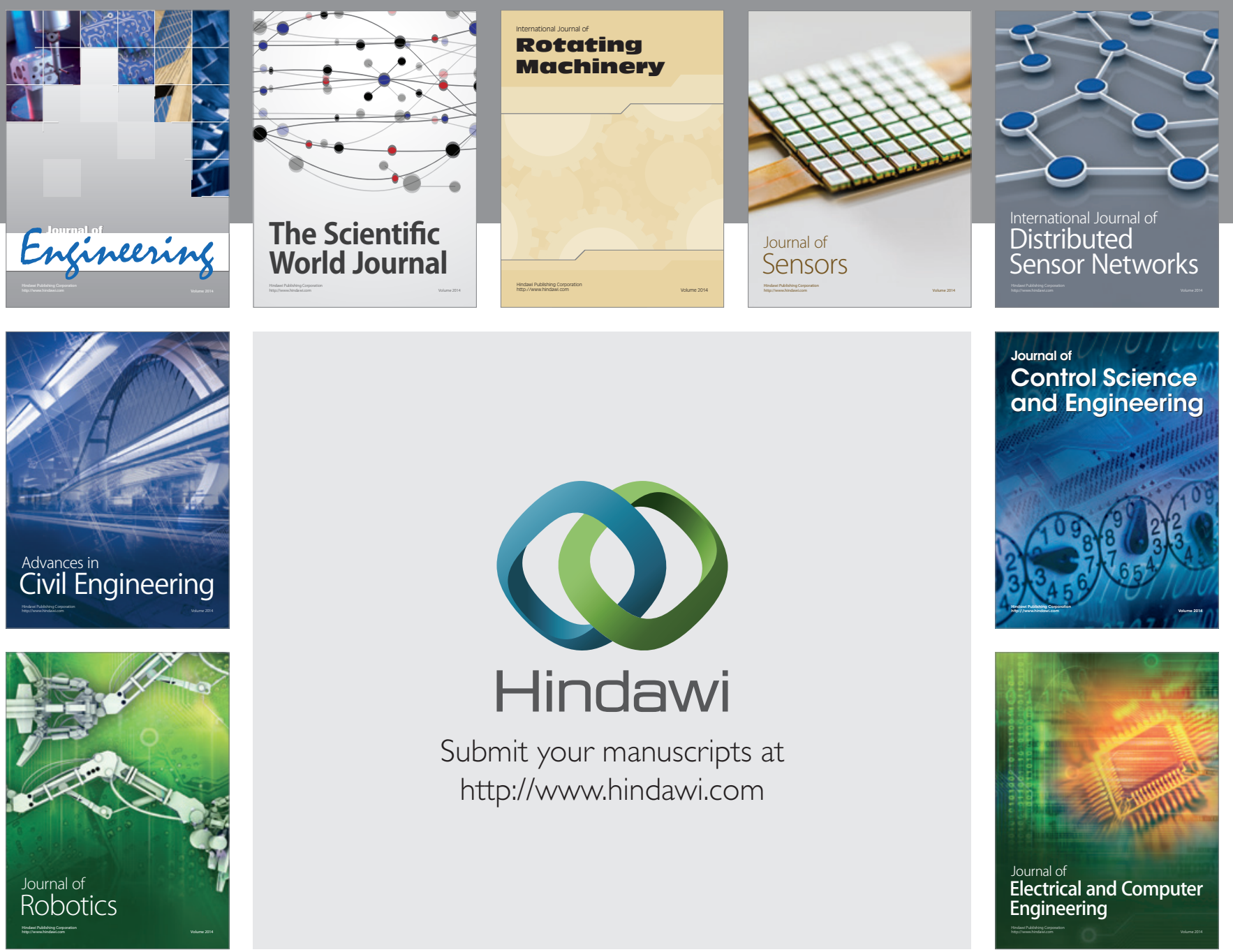

Submit your manuscripts at

http://www.hindawi.com
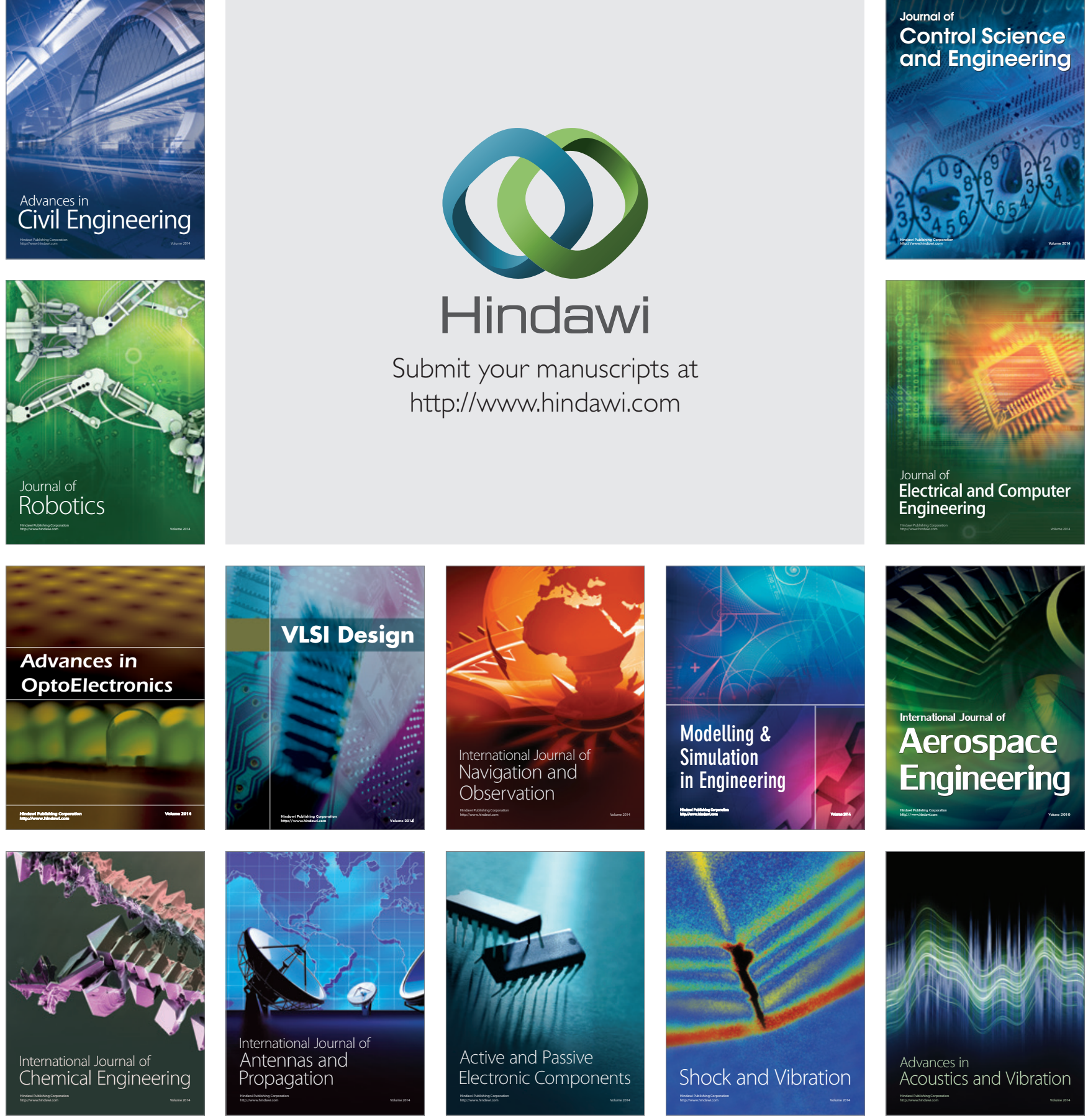